\title{
Validation of diffuse correlation spectroscopy sensitivity to nicotinamide-induced blood flow elevation in the murine hindlimb using the fluorescent microsphere technique
}

Ashley R. Proctor

Gabriel A. Ramirez

Songfeng Han

Ziping Liu

Tracy M. Bubel

Regine Choe 


\title{
Validation of diffuse correlation spectroscopy sensitivity to nicotinamide-induced blood flow elevation in the murine hindlimb using the fluorescent microsphere technique
}

\author{
Ashley R. Proctor, ${ }^{a}$ Gabriel A. Ramirez, ${ }^{a}$ Songfeng Han, ${ }^{b}$ Ziping Liu, ${ }^{a}$ Tracy M. Bubel, ${ }^{c}$ and Regine Choe ${ }^{\mathrm{a}, \mathrm{d}, *}$ \\ aUniversity of Rochester, Department of Biomedical Engineering, Rochester, New York, United States \\ bUniversity of Rochester, Institute of Optics, Rochester, New York, United States \\ 'University of Rochester, Center for Visual Science, Rochester, New York, United States \\ dUniversity of Rochester, Department of Electrical and Computer Engineering, Rochester, New York, United States
}

\begin{abstract}
Nicotinamide has been shown to affect blood flow in both tumor and normal tissues, including skeletal muscle. Intraperitoneal injection of nicotinamide was used as a simple intervention to test the sensitivity of noninvasive diffuse correlation spectroscopy (DCS) to changes in blood flow in the murine left quadriceps femoris skeletal muscle. DCS was then compared with the gold-standard fluorescent microsphere (FM) technique for validation. The nicotinamide dose-response experiment showed that relative blood flow measured by DCS increased following treatment with 500- and 1000-mg/kg nicotinamide. The DCS and FM technique comparison showed that blood flow index measured by DCS was correlated with FM counts quantified by image analysis. The results of this study show that DCS is sensitive to nicotinamide-induced blood flow elevation in the murine left quadriceps femoris. Additionally, the results of the comparison were consistent with similar studies in higherorder animal models, suggesting that mouse models can be effectively employed to investigate the utility of DCS for various blood flow measurement applications. ๑ 2018 Society of Photo-Optical Instrumentation Engineers (SPIE) [DOI: 10.1117/1. JBO.23.3.035006]
\end{abstract}

Keywords: diffuse correlation spectroscopy; fluorescent microspheres; skeletal muscle; blood flow; nicotinamide. Paper 170820R received Dec. 20, 2017; accepted for publication Mar. 6, 2018; published online Mar. 28, 2018.

\section{Introduction}

Nicotinamide is the amide derivative of vitamin $\mathrm{B}_{3} .{ }^{1}$ The clinical utility of nicotinamide has been studied in fields such as dermatology, ${ }^{2}$ neurology, ${ }^{3-6}$ and oncology ${ }^{7}$ to treat various types of skin diseases, to reduce infarction following focal cerebral ischemia or traumatic brain injury (TBI), and to prime hypoxic tumors for radiotherapy, respectively.

The radiosensitizing effect of nicotinamide, in particular, has been widely demonstrated in a variety of tumor models. ${ }^{7-10}$ The mechanism of radiosensitization has been attributed to improved tumor perfusion by reducing transient blood flow fluctuations. ${ }^{7}$ Nicotinamide has been shown to mainly affect tumor blood flow; ${ }^{7,11-13}$ however, it has been shown to affect blood flow in normal tissues as well. In particular, Kelleher and Vaupel ${ }^{14}$ compared red blood cell (RBC) flux measured by laser Doppler using needle probes in rat tumor and skeletal muscle tissues following treatment with $500-\mathrm{mg} / \mathrm{kg}$ nicotinamide. An increase in RBC flux was observed in both tumor and skeletal muscle tissues; however, the increase in skeletal muscle RBC flux was not statistically significant. Although not statistically significant, we hypothesized that the effect of nicotinamide on blood flow in normal tissues could be utilized to test the sensitivity of blood flow imaging modalities, including diffuse correlation spectroscopy (DCS).

*Address all correspondence to: Regine Choe, E-mail: regine_choe@urmc. rochester.edu
DCS is a noninvasive, deep-tissue imaging technique that uses near-infrared light to measure blood flow. ${ }^{15}$ DCS is capable of quantifying blood flow in a variety of tissues, including nonhuman animal and human brain, skeletal muscle, and tumor. ${ }^{15}$ Furthermore, DCS has been validated with other standard methods for measuring blood flow, ${ }^{16-27}$ including the microsphere technique. In the microsphere technique, optical or radiolabeled microspheres are typically injected into the left atrium or ventricle of the heart to ensure homogeneous mixing in the central circulation. In the peripheral circulation, the microspheres become entrapped in the capillaries in direct proportion to the regional blood flow in a tissue. The extraction of $15-\mu \mathrm{m}-$ diameter microspheres is effectively complete, ${ }^{28}$ which means that nearly all of the microspheres become entrapped in the capillaries. By contrast, the extraction of classic diffusible tracers, such as rubidium- $86\left({ }^{86} \mathrm{Rb}\right)$, is incomplete because it is limited by capillary wall permeability, which varies by tissue type. ${ }^{28}$ The microsphere technique has thus become an attractive alternative to diffusible tracers for regional blood flow measurement in various tissue types, including skeletal muscle. ${ }^{29}$

The sensitivity of microspheres to microvascular blood flow has made the microsphere technique a useful tool for the validation of DCS, which is also sensitive to microvascular blood flow. ${ }^{15}$ Zhou et al. $^{30}$ demonstrated the correlation $(R=0.89)$ between relative cerebral blood flow measured by DCS and fluorescent microspheres (FM) in a piglet closed head injury model of TBI $(n=8)$. Mesquita et al. ${ }^{31}$ and Kogler et al. ${ }^{32}$

1083-3668/2018/\$25.00 @ 2018 SPIE 
perturbed spinal cord blood flow in Dorset sheep $(n=10$ and 7 , respectively) by several pharmacological, physiological, and mechanical interventions to induce hypo- and hypertension as well as ischemia. The correlation between relative spinal cord blood flow measured by DCS and stable labeled microspheres (an alternative to optically labeled microspheres) ${ }^{33}$ was $R=0.93$ and 0.70 , respectively.

The aforementioned studies ${ }^{30-32}$ were designed to investigate specific cases of cerebral and spinal hemodynamic insult. They involved higher-order animal models and required specialized systems (e.g., HYGE ${ }^{\mathrm{TM}}$ pneumatic actuator to induce TBI) or surgical procedures (e.g., laminectomy or laminotomy, arterial and venous cannulation, and probe placement under fluoroscopic guidance) to induce the necessary experimental conditions. This study, however, sought to validate DCS as a method to measure blood flow in mice, which are widely used in biomedical research. Additionally, intraperitoneal (IP) injection-a common route of administration in small laboratory animals ${ }^{34}$ - of nicotinamide was investigated as a simple intervention to induce a range of blood flow values to test the sensitivity of DCS. In this study, DCS was employed to continuously measure blood flow in the left quadriceps femoris skeletal muscle of mice with or without nicotinamide treatment. Additionally, the gold-standard FM technique ${ }^{35}$ was employed to validate blood flow index (BFI) quantified by DCS $30 \mathrm{~min}$ after injection.

\section{Materials and Methods}

The first experiment in this study employed DCS to characterize the effect of different doses of nicotinamide on relative blood flow (rBF) in the murine left quadriceps femoris. In the second experiment, DCS was compared to the FM technique.

\subsection{Ethics Statement}

This study was conducted according to a protocol approved by the University Committee on Animal Resources (UCAR) at the University of Rochester. Mice were housed according to the UCAR mouse cage density policy in an animal facility with a 12:12 light-dark cycle. Water and food were available to mice ad libitum.

\subsection{Diffuse Correlation Spectroscopy Instrument}

The DCS instrument comprised a 785-nm-long-coherencelength laser (DL785-120-SO, CrystaLaser, Reno, Nevada), a custom-made probe, and a detection unit containing a fourchannel photon-counting avalanche photodiode (SPCM-AQ4C, Excelitas, Waltham, Massachusetts) and a four-channel correlator board (Flex03OEM, Correlator.com, Bridgewater, New Jersey) (Fig. 1). The correlator board calculates the normalized intensity temporal autocorrelation function of detected light, from which BFI is derived using the semi-infinite solution to the correlation diffusion equation. ${ }^{15}$

The custom-made probe (Fiberoptic Systems, Inc., Simi Valley, California) contained one $200-\mu$ m-diameter multimode fiber for the light source and four 5.6- $\mu \mathrm{m}$-diameter singlemode fibers for the detectors (Fig. 1). Source-detector separations were 2.89, 3.60, 4.28, and $5.00 \mathrm{~mm}$. A micromanipulator and a linear translational stage were used to control the positioning of the probe on the lateral hindlimb for the single point measurement taken just above the femoral diaphysis (e.g.,

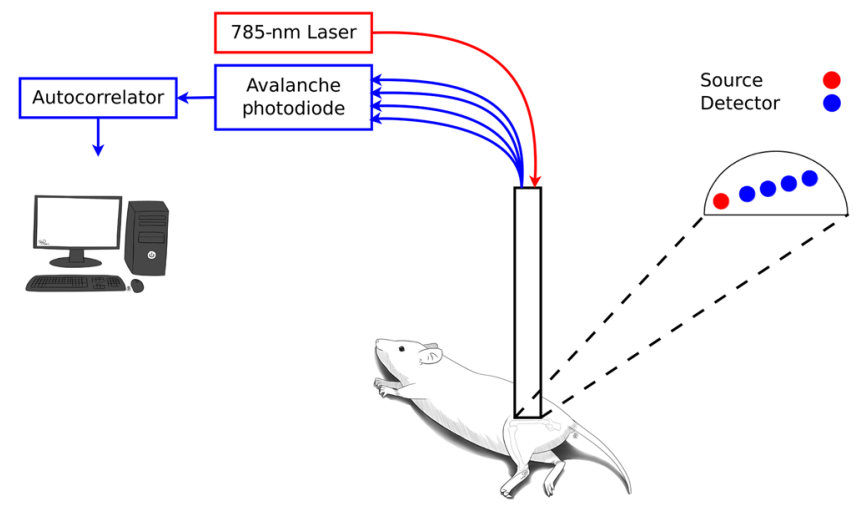

Fig. 1 DCS instrument and probe placement. The probe was placed on the lateral hindlimb over the left quadriceps femoris. The multimode fiber (the leftmost fiber in the probe) delivered near-infrared light from the 785-nm-long-coherence-length laser to the tissue surface. Light that propagated through the tissues was detected by four single-mode fibers at source-detector separations of $2.89,3.60,4.28$, and $5.00 \mathrm{~mm}$ and relayed to the four-channel photon-counting avalanche photodiode. Normalized intensity temporal autocorrelation functions were calculated by the correlator board and saved in the computer.

approximately in the middle of the upper hindlimb) on the left quadriceps femoris skeletal muscle (Fig. 1).

\subsection{Nicotinamide Dose-Response Experiment}

In this experiment, 6- to 7-week-old female BALB/cByJ mice (The Jackson Laboratory, Bar Harbor, Maine) were randomized into three groups that received $200-\mu \mathrm{L}$ phosphate-buffered saline (PBS) (vehicle control), 500-, or $1000-\mathrm{mg} / \mathrm{kg}$ nicotinamide. For the purpose of this study, 500- and $1000-\mathrm{mg} / \mathrm{kg}$ nicotinamide are henceforth referred to as the half and full doses, respectively.

Nicotinamide was prepared in $200-\mu \mathrm{L}$ PBS immediately before the start of the experiment. Anesthesia was induced and maintained with isoflurane (2\%), and body temperature was maintained by a water-circulating thermal pad. Mice were placed in the right lateral recumbent position, and the fur on the left hindlimb was removed by electric clipper and depilatory cream (Nair ${ }^{\mathrm{TM}}$, Church \& Dwight Co., Inc., Ewing, New Jersey) immediately before the experiment.

The DCS probe was aligned such that the source and detectors were positioned over the left quadriceps femoris as shown in Fig. 1. A permanent marker was used to mark the probe position on the skin for repeatable placement following the intervention. Baseline DCS measurements were acquired for $5 \mathrm{~min}$, with each autocorrelation function integrated for $2 \mathrm{~s}$. Then, the probe was removed and mice received the vehicle, half dose, or full dose nicotinamide by IP injection. The probe was then replaced, and postintervention DCS measurements were acquired for an additional $30 \mathrm{~min}$. Ambient and body temperatures were monitored to ensure that there were no drastic changes throughout the experiment.

\subsection{DCS and Fluorescent Microsphere Technique Comparison}

For the DCS and FM technique comparison, DCS was employed to quantify BFI before and after intervention. FM injection followed the 30-min postintervention DCS 
(a)

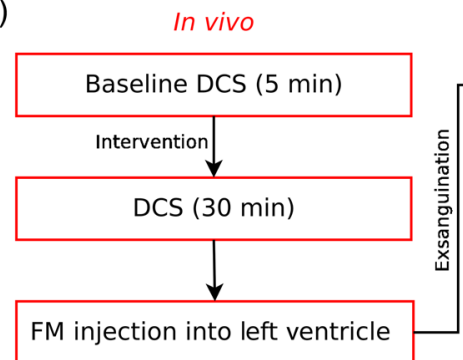

Postmortem

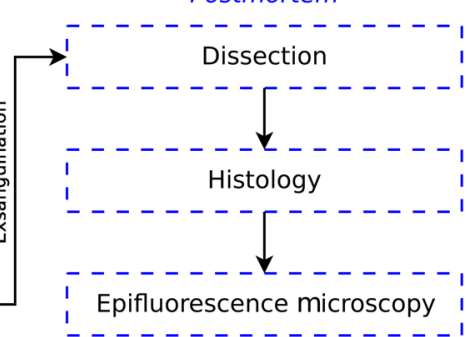

(b)

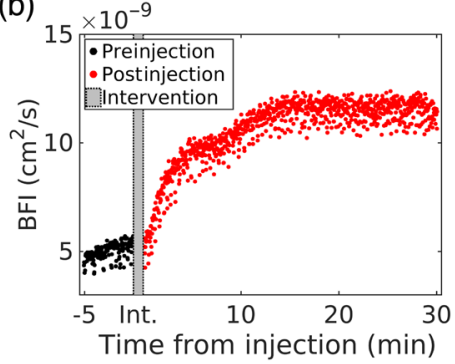

Fig. 2 DCS and FM technique comparison. (a) Flow chart illustrating the steps of the DCS and FM technique comparison. DCS blood flow measurement preceded the FM injection, which was a terminal procedure in this study. (b) Representative BFI $\left(\mathrm{cm}^{2} / \mathrm{s}\right)$ temporal changes in the left quadriceps femoris of a mouse treated with $1000-\mathrm{mg} / \mathrm{kg}$ nicotinamide.

measurements. Part of Fig. 2(a) summarizes the in vivo steps of the DCS and FM technique comparison experimental design, and Fig. 2(b) shows the representative in vivo BFI temporal changes following treatment with full dose nicotinamide. The postmortem procedures outlined in Fig. 2(a) were then performed following exsanguination.

\subsubsection{DCS blood flow measurement}

In this experiment, 6- to 12-week-old female BALB/cByJ mice were prepared, and pre- and postintervention blood flow were measured by DCS as described in Sec. 2.3.

Control mice did not receive a vehicle injection to minimize potential blood flow perturbation. Note that the BFI difference between control mice with and without vehicle injection was not statistically significant (Appendix A). Treatment mice received full dose nicotinamide to achieve the maximum blood flow change compared to the no-vehicle control mice to test the sensitivity of DCS and the FM technique. Both no-vehicle control and full dose nicotinamide mice were perturbed similarly: picked up (with or without injection), repositioned, and probe replaced.

\subsubsection{Fluorescent microsphere blood flow measurement}

The FM injection protocol was based on Serrat. ${ }^{36}$ After the 30-min DCS blood flow measurement, the skin and superficial muscle layer overlying the thorax were incised to visualize the ribs. While the skin and muscle were being surgically reflected (i.e., moved away from the ribs), the FMs were prepared for injection by vortexing for $15 \mathrm{~s}$, sonicating for $5 \mathrm{~min}$, and vortexing again for $15 \mathrm{~s}$ to agitate the microspheres before being loaded into a syringe attached to a $27 \mathrm{G}$ needle. Once the syringe was loaded, the hemithoracotomy (i.e., incision of the intercostal muscles to open the thoracic cavity) was performed and an Agricola retractor was inserted between the ribs to expose the thoracic viscera. To prevent drastic hemodynamic perturbation due to asphyxiation, the FMs were injected into the left ventricle of the heart immediately after opening the thoracic cavity. A volume of $150 \mu \mathrm{L}$ containing $150,000 \mathrm{FMs}$ was injected and the microspheres were allowed to circulate for $60 \mathrm{~s}$. After $60 \mathrm{~s}$, the mouse was euthanized by exsanguination.

Yellow-green FluoSpheres ${ }^{\mathrm{TM}} \quad$ (Invitrogen $^{\mathrm{TM}}$ Molecular Probes $^{\mathrm{TM}}$, Carlsbad, California) with excitation and emission wavelengths of 505 and $515 \mathrm{~nm}$, respectively, were used. The FMs were $15 \mu \mathrm{m}$ in diameter and were chosen because microspheres of this size are effectively $100 \%$ extracted, ${ }^{28}$ which means that nearly all of the microspheres become entrapped in the capillaries.

\subsection{Histology and Microscopy}

Each left hindlimb or left quadriceps femoris was harvested, fixed in $4 \%$ paraformaldehyde at room temperature for $2 \mathrm{~h}$, and cryoprotected in $15 \%$ and $30 \%$ sucrose, respectively, at $4^{\circ} \mathrm{C}$ overnight. During fixation, each left quadriceps femoris was either left attached to the femur or loosely tied to a dowel with nylon suture to preserve its natural length. Once fixed and cryoprotected, the left quadriceps femora were removed from the femur or dowel for frozen sectioning. The left quadriceps femora were mounted on the stage of a Microm HM 400R manual sliding microtome (Microm Laborgeräte $\mathrm{GmbH}$, Walldorf, Germany) in the same orientation as in situ during the DCS measurements; thus, the left quadriceps femora were frozen sectioned into $100-\mu$ m-thick tissue sections along the sagittal plane in the lateral to medial direction.

The FMs were visualized at $2 \times$ magnification by epifluorescence microscopy (Olympus BX53 Upright Microscope, Olympus Corporation, Tokyo, Japan), and images were obtained using the software Neurolucida (MBF Bioscience, Williston, Vermont), which controlled the translation of the motorized stage. Minimal background illumination (using the substage illuminator) was employed during imaging to visualize the tissue sections, which were very dark compared to the bright yellow-green FMs. For each section imaged, tissue section area was determined, and FMs were quantified by image analysis, as described in Sec. 2.6.

\subsection{Data Analysis}

\subsubsection{Blood flow index extraction and relative blood flow calculation}

Each BFI of the DCS data was calculated using the solution for the correlation diffusion equation of homogeneous medium with semi-infinite boundary conditions. ${ }^{15}$ Typically, BFI is determined by fitting the analytical model to the measured intensity temporal autocorrelation function for a single source-detector pair; however, this method often results in different BFI for each source-detector pair. The differing BFI values are either treated as independent values or averaged to determine bulk BFI. In this study, however, the source-detector separations stated in Sec. 2.2 were fitted concurrently to yield a single BFI for a homogeneous medium using the method adapted from Farzam and Durduran. ${ }^{37}$ The analytical solution was 
simultaneously fitted to the measured intensity temporal autocorrelation function for each source-detector pair using the penalty function

$\chi^{2}=\sum_{i=1}^{4} \sum_{\tau} \frac{\left(g_{2 ; \text { measured }, i, \tau}-g_{2 ; \text { analytical }, i, \tau}\right)^{2}}{g_{2 ; \text { measured }, i, \tau}}$,

where $i$ is the source-detector pair, $\tau$ is the delay time, $g_{2 \text {;measured }}$ is the measured intensity temporal autocorrelation function, and $g_{2 \text {;analytical }}$ is the intensity temporal autocorrelation function of the analytical model. This method implies a weighing function to determine the average bulk BFI measured by the sourcedetector pairs and provides a stable fitting result. The optical parameters were assumed to be constant with an absorption coefficient of $0.1 \mathrm{~cm}^{-1}$ and reduced scattering coefficient of
$8.0 \mathrm{~cm}^{-1} \cdot{ }^{38} \mathrm{rBF}$ was calculated by normalizing BFI with the average baseline BFI.

\subsubsection{Tissue section image stitching}

Multiple images were needed to capture each tissue section as shown in Fig. 3(a). The images were stitched together using an automated algorithm written in MATLAB ${ }^{\mathrm{TM}}$, which relies on identifying speeded up robust features (SURFs). ${ }^{39}$ The images must be taken with low incident illumination (using the substage illuminator) to prevent saturating the fluorescence intensity of the FMs; otherwise, the background and microspheres have similar intensities, rendering microsphere identification extremely difficult. The low incident illumination precludes reliable identification of SURFs. To rectify this, the algorithm first converts the raw images to grayscale and then rescales the pixel
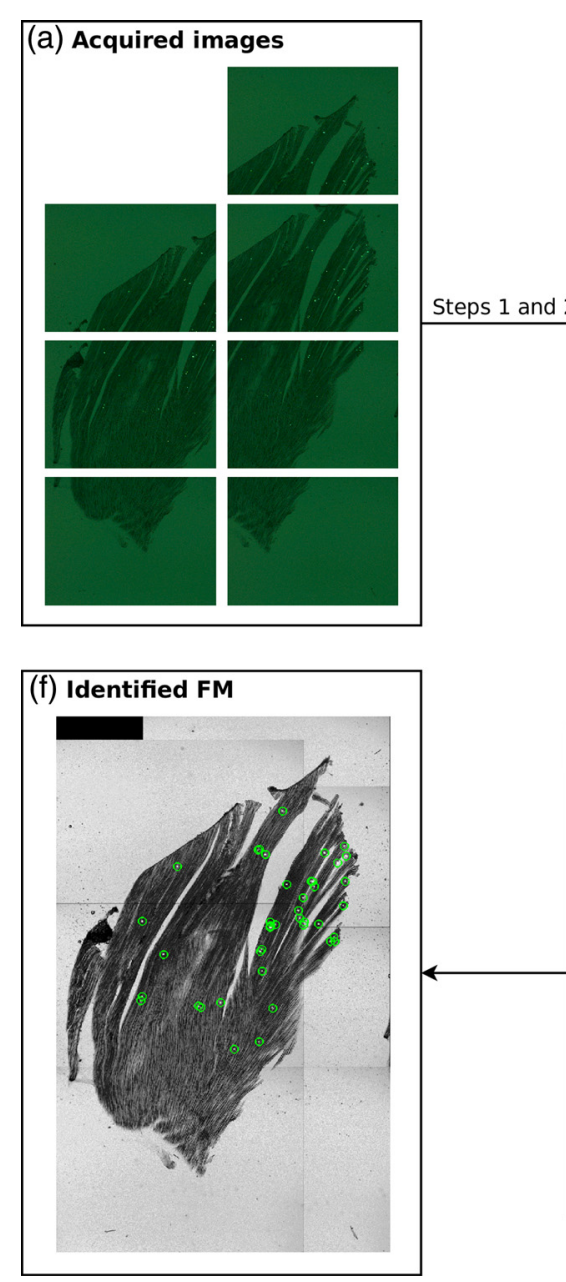

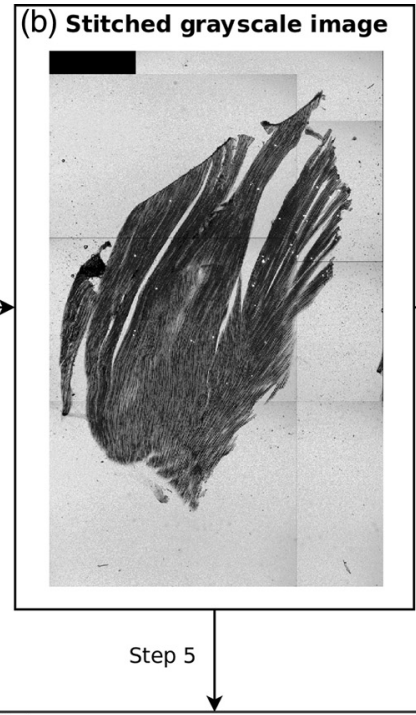

(e) FM identification

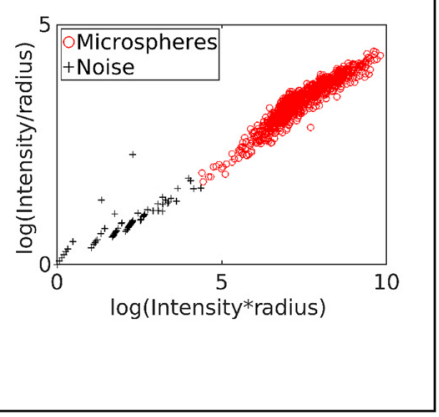

(c) Inverted grayscale image

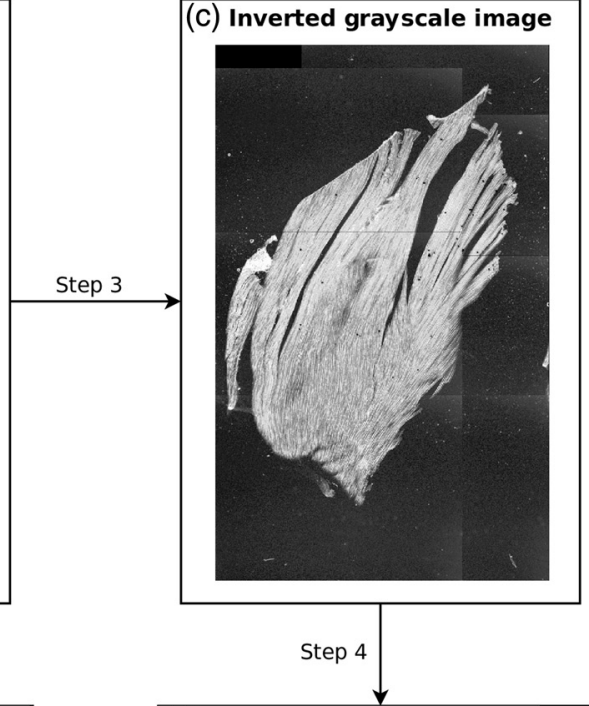

(d) Tissue area identification
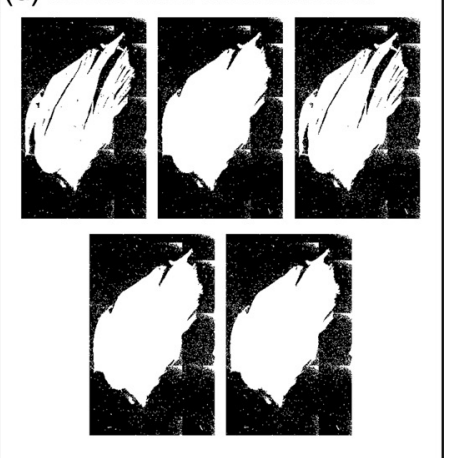

Fig. 3 Image analysis process. (a) Acquired images of a left quadriceps femoris tissue section. Note that the acquired images were brightened using GNU Image Manipulation Program only for the purpose of presentation. The raw acquired images, which are analyzed, appear nearly black with the exception of a few small yet bright yellow-green spots (i.e., FMs). In step 1, the acquired images are converted to grayscale images. In step 2, the grayscale images are stitched using SURFs, yielding (b). In step 3, the stitched grayscale image is inverted, yielding (c). In step 4, the inverted grayscale image is converted to binary images of the optimal tissue section area mask with postthresholding adjustments, yielding (d), which are then averaged. In step 5, FMs are identified using $k$-means clustering, yielding (e), which represents all of the FM in an entire left quadriceps femoris. Intensity-radius pairs of pixels are grouped and separated into noise (black plus signs) and FM (red open circles). (f) An example of identified FM (green open circles) in the stitched grayscale image of the tissue section as a result of the image analysis. In (b), (c), (d), and (f), the black area in the upper left corner of the stitched images is the background imparted by Neurolucida, which is not included in the image analysis. 
intensities such that the lowest $2 \%$ pixel intensities are set to zero and the highest $2 \%$ pixel intensities are saturated.

The algorithm identifies SURFs in the grayscale images, identifies matching features between each combination of images, and stitches the combination with the most matching features. If there is a tie for most matching features, a tied combination is chosen at random. Ties occur in $<0.1 \%$ of cases, and random selection does not affect the reproducibility of the image stitching. The two matched images are then dropped and replaced with the stitched image. SURFs are calculated for the newly stitched image, and the process is repeated until only one fully stitched image remains as shown in Fig. 3(b).

\subsubsection{Tissue area identification}

To identify the tissue area, the stitched grayscale image is inverted as shown in Fig. 3(c) and then repeatedly converted to a binary image using a threshold that varies from no thresholding to saturation. The total area for each binary image is determined by calculating the number of pixels above threshold. As the threshold is increased, the area decreases, resulting in an L-curve when plotted against each other. The threshold and area are then normalized to have a maximum value of one. The threshold-area pair with the shortest distance from the origin is taken as the ideal threshold and the resulting binary image is accepted as the area. Threshold values below this pairing increase false-positive area pixels, and threshold values above this pairing increase false-negative area pixels.

Tissue variations caused by histological artifacts (e.g., folding, tearing, and heterogeneous tissue section thickness) or lack of variation between tissue and background can lead to underestimating tissue area. To address these variations, various postthresholding adjustments (i.e., unaltered, dilated, filled, dilated then filled, or filled then dilated) are made as shown in Fig. 3(d). Then, the estimated tissue areas yielded from the postthresholding adjustments are averaged.

\subsubsection{Fluorescent microsphere identification}

To identify the FMs, a threshold is applied to the modified grayscale image such that only the highest $2 \%$ of pixel intensities are retained, whereas all others are set to zero. The remaining pixels are grouped, and their corresponding intensities and radii are estimated. ${ }^{40}$ For the imaging configuration in this study, FMs tend to have radii exceeding 2 pixels, with collective pixel intensities exceeding 500, whereas noise tends to have radii below 2 pixels, with intensities below 500. Rather than relying on these rules of thumb, the intensity-radius pairs are further separated using the empirical transform

$$
\begin{aligned}
& X=\log [\text { Intensity } \times \text { Radius } / \min (\text { Intensity } \times \text { Radius })] \\
& Y=\log [\text { Intensity } / \text { Radius } / \min (\text { Intensity } / \text { Radius })]
\end{aligned}
$$

The intensity-radius pairs are pooled for the entire left quadriceps femoris and are separated into noise and FM clusters using $k$-means clustering. ${ }^{41}$ Pooling data is necessary because some tissue sections do not have FMs, making clustering impossible. The final FM clusters for an entire left quadriceps femoris are shown in Fig. 3(e). The FMs identified by the algorithm are shown for a single section in Fig. 3(f).
Table 1 Number of mice per group after exclusion. Note that there was no overlap between the two experiments.

\begin{tabular}{lllll} 
& \multicolumn{3}{c}{ Nicotinamide } & \\
\cline { 2 - 4 } Experiment & None & Half & Full & FM \\
\hline $\begin{array}{l}\text { Nicotinamide dose-response } \\
\text { experiment }\end{array}$ & $n=9$ & $n=10$ & $n=20$ & No \\
$\begin{array}{l}\text { DCS and FM technique } \\
\text { comparison }\end{array}$ & $n=9$ & NA & $n=8$ & Yes \\
\hline
\end{tabular}

Note: NA is not applicable.

\subsubsection{Exclusion criteria and normalization}

For the nicotinamide dose-response experiment, one vehicle control mouse out of 10 was excluded because the mouse woke up during the DCS measurements.

For the DCS and FM technique comparison, one no-vehicle control and three full dose nicotinamide mice out of 21 total mice were excluded because there were very few or no FMs in their left quadriceps femora. Table 1 shows the number of mice per group by experiment after exclusion.

For each muscle, the FM counts were aggregated and then normalized using the total tissue area. Total tissue area was used to normalize the FM counts because tissue sample weight was not known for left quadriceps femora that were left attached to the femur during fixation $(n=6)$. In a separate analysis (not shown), the known weights $(n=11)$ of the left quadriceps femora were highly correlated with the tissue area $(R=0.95, p \ll 0.01)$.

\subsubsection{Statistical analysis}

Statistical analysis of the averaged final $5 \mathrm{~min}$ of the nicotinamide dose-response experiment was performed using MATLAB ${ }^{\mathrm{TM}}$. A one-way analysis of variance was performed to compare the effect of nicotinamide dose on rBF in three groups: vehicle control $(n=9)$, half dose nicotinamide $(n=10)$, and full dose nicotinamide $(n=20)$. There was a significant effect of nicotinamide dose on $\mathrm{rBF}$ at the $p \ll 0.01$ level. Therefore, post-hoc analysis was conducted using the student's unpaired two-sample $t$-test to compare the three groups with Bonferroni correction, for a total of three comparisons.

\section{Results}

\subsection{Nicotinamide-Induced Blood Flow Elevation}

The results of the nicotinamide dose-response experiment (described in Sec. 2.3) are shown in Fig. 4 as the rBF for the vehicle control $(n=9)$, half dose $(500-\mathrm{mg} / \mathrm{kg}$ nicotinamide, $n=10)$, and full dose (1000-mg/kg nicotinamide, $n=20$ ) groups.

Each point in Fig. 4(a) represents the group-averaged temporal $\mathrm{rBF}$ of all mice in a group at 20-s intervals, and the error bar represents the standard error of the mean (SEM). Baseline $\mathrm{rBF}$ was relatively steady for all mice, which is expected and suggests that the DCS measurements were not affected by external environmental factors, such as ambient temperature. $\mathrm{rBF}$ increased over time for the half and full dose treatment groups, but did not change for the vehicle control group.

The group-averaged $\mathrm{rBF}$ at $30 \mathrm{~min}$ is shown in Fig. 4(b). $\mathrm{rBF}$ at $30 \mathrm{~min}$ was $1.03 \pm 0.22$ (mean \pm standard deviation), 
(a)

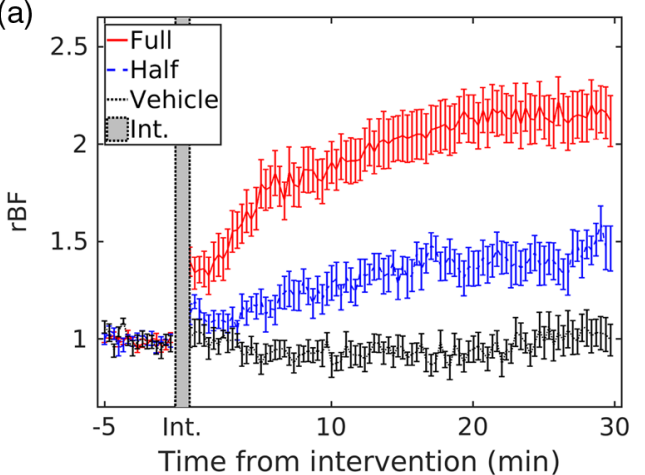

(b)

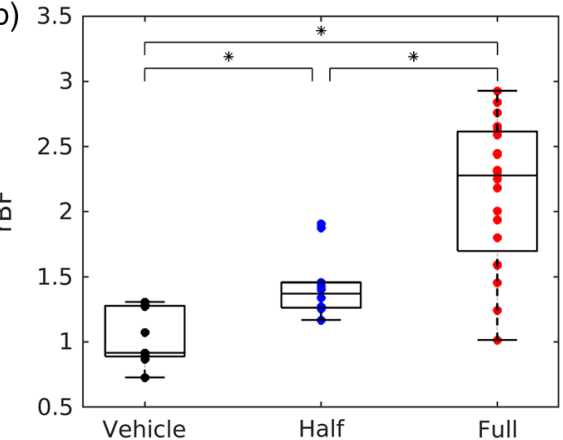

Fig. 4 Nicotinamide-induced blood flow changes measured by DCS. (a) Group-averaged temporal changes in blood flow normalized with respect to the baseline (rBF) are shown for all groups. The full dose nicotinamide group shows the greatest temporal change in rBF. Only every 10th measurement $(\Delta t=20 \mathrm{~s})$ is shown for clarity. (b) Average rBF of the last $5 \mathrm{~min}$ of the DCS measurements, where vehicle is vehicle control, half is $500-\mathrm{mg} / \mathrm{kg}$ nicotinamide, and full is $1000-\mathrm{mg} / \mathrm{kg}$ nicotinamide. * denotes $p<0.017$.

$1.44 \pm 0.26$, and $2.15 \pm 0.55$ for the vehicle control, half dose, and full dose group, respectively. rBF changes among all groups were significantly different from each other (see Table 2).

\subsection{Comparing the Control and Treatment Effects on $\mathrm{BFI}$}

Table 2 shows the results of the $t$-tests comparing the averaged final $5 \mathrm{~min}$ of the nicotinamide dose-response experiment. The

Table 2 Statistical analysis results.

\begin{tabular}{lcccc} 
Comparison & $\begin{array}{c}\text { Difference in } \\
\text { group means }\end{array}$ & $p$ value & $\begin{array}{c}95 \% \mathrm{Cl} \\
\text { lower } \\
\text { bound }\end{array}$ & $\begin{array}{c}95 \% \mathrm{Cl} \\
\text { upper } \\
\text { bound }\end{array}$ \\
\hline Control versus half & 0.34 & $0.001^{*}$ & 0.16 & 0.52 \\
Control versus full & 0.72 & $\ll 0.001^{*}$ & 0.50 & 0.94 \\
Half versus full & 0.38 & $<0.001^{*}$ & 0.17 & 0.58 \\
\hline
\end{tabular}

Note: $\mathrm{Cl}$ is the confidence interval.

${ }^{\star}$ Bonferroni corrected $p<0.017$

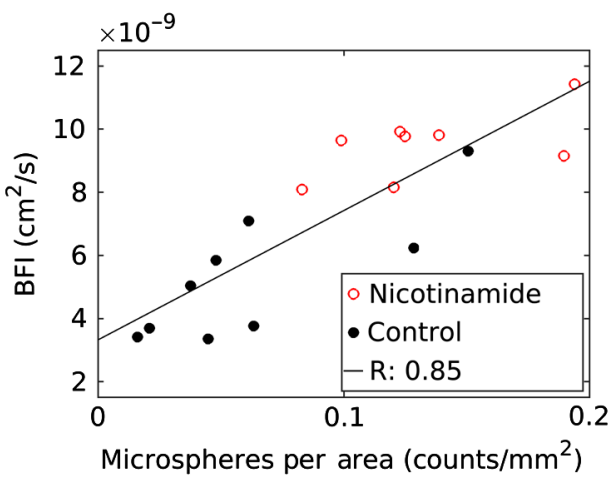

Fig. 5 Correlation between DCS and the FM technique. As BFI $\left(\mathrm{cm}^{2} / \mathrm{s}\right)$ increases, FM counts $/ \mathrm{mm}^{2}$ increases. The no-vehicle control mice (black closed circles) tended to display lower BFI and FM counts compared to mice treated with $1000-\mathrm{mg} / \mathrm{kg}$ nicotinamide (red open circles). There were, however, a few control mice with blood flow values similar to those of treatment mice. results show that all groups were significantly different from each other $(p \ll 0.01)$. With the Bonferroni correction, the results are still significant because the adjusted $\alpha=0.05 / 3$ for three comparisons is 0.017 .

\subsection{DCS and Fluorescent Microsphere Technique Correlation}

Figure 5 shows the results of the DCS and FM technique comparison experiment described in Sec. 2.4 as the correlation between the two blood flow measurement methods. The results show a moderate correlation $(R=0.85, p \ll 0.01)$ between BFI $\left(\mathrm{cm}^{2} / \mathrm{s}\right)$ and number of FMs per area (counts $\left./ \mathrm{mm}^{2}\right)$ for each left quadriceps femoris.

\section{Discussion}

In this study, the FM technique was employed for comparison with DCS to measure murine skeletal muscle blood flow. To test the sensitivity of both methods, nicotinamide was used to modulate skeletal muscle blood flow based on Kelleher and Vaupel, ${ }^{14}$ in which laser Doppler needle probes directly measured skeletal muscle RBC flux. The results of this study show that both DCS and the FM technique are sensitive to nicotinamide-induced blood flow elevation in the left quadriceps femoris of mice.

DCS blood flow measurements are comprised of signals from multiple types of tissues (i.e., skin and skeletal muscle), and the skin versus skeletal muscle signal contribution has not been resolved in this study. Yet, DCS measurements showed a significant increase in left quadriceps femoris $\mathrm{rBF}$ in response to $500-\mathrm{mg} / \mathrm{kg}$ nicotinamide. This was similar to the trend observed by Kelleher and Vaupel despite the discrepancy in statistical significance, which may be attributable to the different animal models (rat versus mouse) and mode of nicotinamide administration (10-min infusion versus bolus injection) employed in each study. Furthermore, DCS-derived BFI agreed with FM counts in the left quadriceps femora of mice treated with $1000-\mathrm{mg} / \mathrm{kg}$ nicotinamide. Thus, DCS reveals similar trends as techniques that directly measure skeletal muscle blood flow. One possible explanation is that skin and skeletal muscle may have similar blood flow. Another possible explanation is that skin has a smaller contribution to the DCS signal compared to skeletal muscle in this measurement configuration. In the future, the contribution of each tissue to the bulk blood flow signal measured by DCS 
can be investigated by measuring both skin and skeletal muscle blood flow using the FM technique.

Earlier studies used alternative blood flow measurement techniques, such as ${ }^{86} \mathrm{Rb}$ extraction, ${ }^{42}$ to investigate the effect of nicotinamide on blood flow in various types of tissues. Hirst et al. ${ }^{43}$ and Honess and Bleehen ${ }^{44}$ measured cardiac output distribution (COD) and relative tissue perfusion (RTP), respectively, in murine tumor and normal tissues, including skeletal muscle. Following 1000-mg/kg nicotinamide treatment, Hirst et al. and Honess and Bleehen found a reduction in normal skeletal muscle COD and RTP, respectively. Unlike the deposition of $15-\mu \mathrm{m}$ microspheres, however, ${ }^{86} \mathrm{Rb}$ extraction is not complete. ${ }^{45,46}$ Furthermore, ${ }^{86} \mathrm{Rb}$ extraction is low when flow is high. ${ }^{46}$ In this study, $1000-\mathrm{mg} / \mathrm{kg}$ nicotinamide significantly increased skeletal muscle rBF by over $200 \%$ as measured by DCS, which presents a very high flow condition. It is, therefore, possible that ${ }^{86} \mathrm{Rb}$ extraction underestimated skeletal muscle blood flow in the aforementioned studies.

The results of this study show that DCS is sensitive to a range of blood flow values induced by $500-$ and $1000-\mathrm{mg} / \mathrm{kg}$ nicotinamide administered intraperitoneally. This is useful to the study of DCS sensitivity because the IP route of administration is accessible in mice and technically simple to perform. Furthermore, the correlation $(R=0.85)$ between DCS and the FM technique in this study was relatively consistent with the results of previous studies ${ }^{30-32}$ that employed both methods to measure $\mathrm{rBF}$ in higher-order animal models, such as piglet $(R=0.89)$ and Dorset sheep $(R=0.93$ and 0.70$)$. This suggests that mice, which are widely used in biomedical research, can be used in DCS validation studies. Although absolute blood flow measurement in mice is technically challenging due to sizerelated concerns (e.g., reference blood sample withdrawal), ${ }^{29}$ multiple injections of differently labeled microspheres are possible. ${ }^{29,47,48}$ Therefore, rBF validation can be achieved by modifying the FM injection protocol through a carotid cannula, ${ }^{29,47,48}$ for example, to sustain mice for multiple microsphere measurements.

Limitations of the current study design may have contributed to the results of the DCS and FM technique comparison. FM injection into the left ventricle of the heart is technically challenging, and it is not possible to precisely control the injection rate among mice without using a preset syringe pump. This could be problematic because adequate mixing in the circulation is critical to the validity of the FM technique. ${ }^{28}$ In the future,

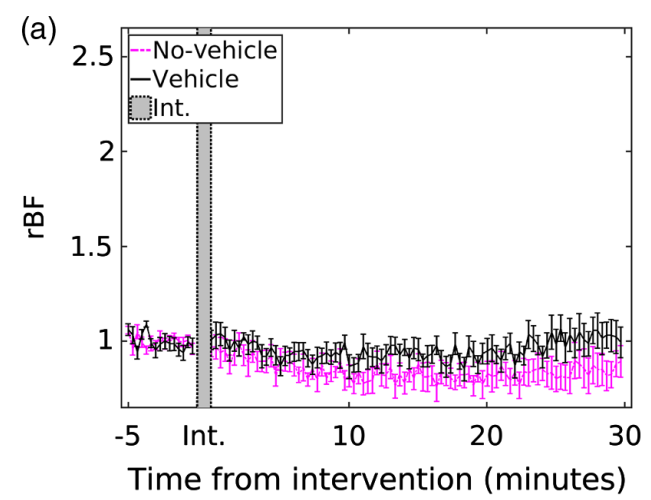

analyzing the kidneys to assess microsphere mixing in the circulation will provide a measure of the validity of the FM technique. Additionally, physiological monitoring (e.g., heart and respiratory rates) was not employed during the DCS measurements and FM injection. It is possible that slightly different hemodynamic signatures were captured by DCS and the FM technique for two reasons. First, the FM injection did not occur until at least 5 min after the DCS measurements due to the preparation of the FMs. During these $5 \mathrm{~min}$, it is possible that BFI was not stable for some mice. Second, the mice were not intubated prior to hemithoracotomy ${ }^{36}$ therefore, mice were asphyxiated during the microsphere injection and circulation period. The effect was, however, minimized by performing the injection immediately following the hemithoracotomy.

\section{Conclusions}

Despite the limitations, this study clearly demonstrated that DCS is sensitive to nicotinamide-induced blood flow elevation in the murine hindlimb. In the future, the FM protocol will be modified to allow for $\mathrm{rBF}$ validation. In addition to tissues of interest, such as skeletal muscle and bone, the kidneys will be analyzed to assess the adequacy of microsphere mixing. The spatial distribution of blood flow is of interest. Therefore, the origin of the blood flow signals will be investigated by first developing a reproducible method to coregister the threedimensionally rendered microscopic images and the threedimensional BFI distribution determined by diffuse correlation tomography (DCT), an imaging analog of DCS. Then, the number of microspheres at different tissue depths will be correlated with the BFI from DCT at the corresponding depths. Tissue optical clearing and higher resolution confocal or multiphoton microscopy may improve FM detection.

Ultimately, DCS and DCT will be validated for skeletal muscle and bone blood flow monitoring in murine bone fracture and graft models to characterize blood flow, which is critical to the bone healing process. ${ }^{49}$

\section{Appendix A}

\section{A1 Comparison of No-Vehicle and Vehicle Control Groups}

Figure 6 shows the rBF for the no-vehicle control group $(n=9)$ from Sec. 2.4 and vehicle control group $(n=9)$ from Sec. 2.3.

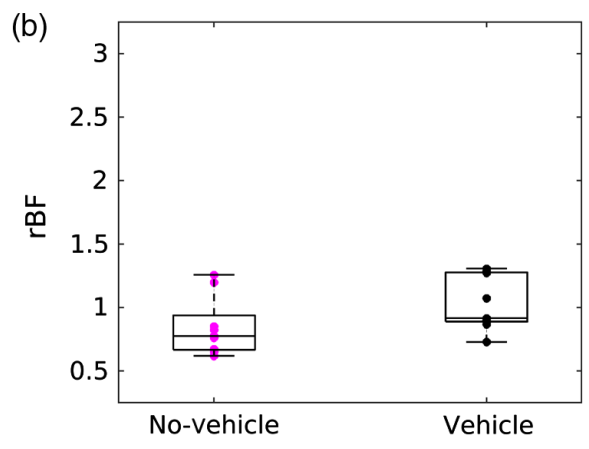

Fig. 6 rBF of the control groups with or without vehicle injection. (a) Group-averaged temporal changes in blood flow normalized with respect to the baseline (rBF) are shown for both control groups. Only every 10th measurement is shown for clarity. (b) Average rBF of the last $5 \mathrm{~min}$ of the DCS measurement, where no-vehicle is no-vehicle control and vehicle is vehicle control. 

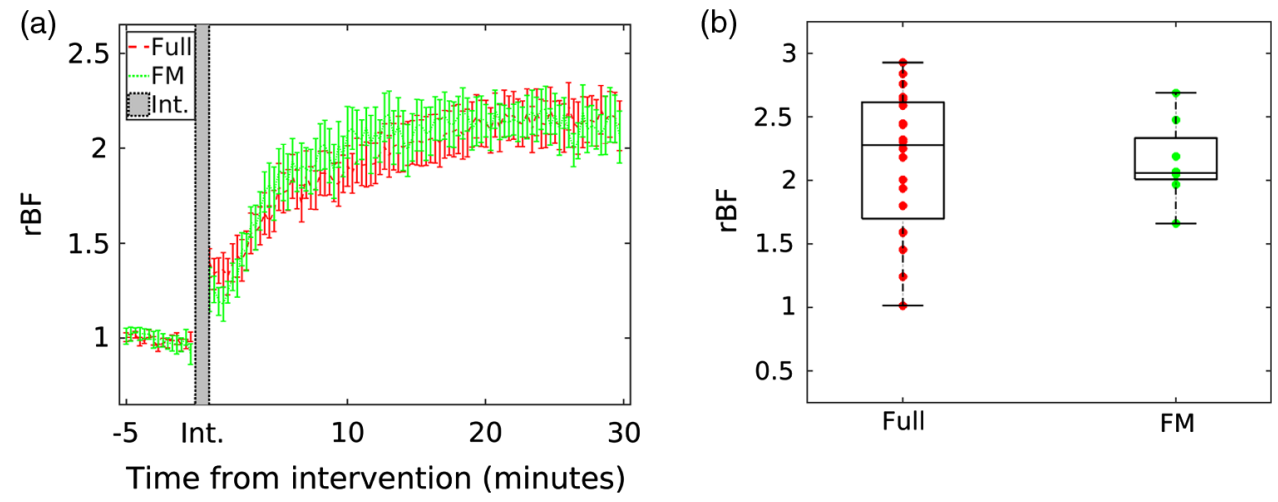

Fig. 7 rBF of the full dose nicotinamide groups. (a) Group-averaged temporal changes in blood flow normalized with respect to the baseline (rBF) are shown for both full dose nicotinamide groups. Only every 10th measurement is shown for clarity. (b) Average rBF of the last 5 min of the DCS measurement, where full is full dose nicotinamide from the nicotinamide dose-response experiment and FM is full dose nicotinamide from the DCS and FM comparison experiment.

Each point in Fig. 6(a) represents the group-averaged temporal $\mathrm{rBF}$ of all mice in a group at 20-s intervals, and the error bar represents the SEM. The group-averaged $\mathrm{rBF}$ at $30 \mathrm{~min}$ is shown in Fig. 6(b).

Statistical analysis of the vehicle injection effect was done using the student's two-sample unpaired $t$-test to compare the no-vehicle control and vehicle control groups. There was no statistically significant difference between the two control groups $(p=0.08)$, which suggests that the vehicle injection had no effect on the BFI during the final 5 min of DCS measurements.

\section{A2 Comparison of Full Dose Nicotinamide Groups}

Figure 7 shows the rBF for the full dose nicotinamide group $(n=20)$ from Sec. 2.3 and full dose nicotinamide group $(n=8)$ from Sec. 2.4. Each point in Fig. 7(a) represents the group-averaged temporal $\mathrm{rBF}$ of all mice in a group at 20-s intervals and the error bar represents the SEM. The group-averaged $\mathrm{rBF}$ at $30 \mathrm{~min}$ is shown in Fig. 7(b).

Statistical analysis of the FM injection effect was done using the student's two-sample unpaired $t$-test to compare the two full dose nicotinamide groups, and there was no statistically significant difference between the groups $(p=0.82)$.

\section{Disclosures}

The authors have no conflicts of interest to disclose.

\section{Acknowledgments}

The authors thank Don Henderson for sharing his expertise in skeletal muscle histology, Ryan Dawes, PhD, for sharing his expertise in epifluorescence microscopy, Mackenzie Harris for assisting with microscopy, and Rejune Choe for illustrations. This study was funded by the National Science Foundation (Award No. CBET 1507384) and the National Institute of Health (NIAMS Award No. R01 AR071363). The funders had no role in the study design, data collection and analysis, decision to publish, or preparation of the paper.

\section{References}

1. J. R. DiPalma and W. S. Thayer, "Use of niacin as a drug," Anпи. Rev. Nutr. 11, 169-187 (1991).
2. H. M. Rolfe, "A review of nicotinamide: treatment of skin diseases and potential side effects," J. Cosmet. Dermatol. 13(4), 324-328 (2014).

3. I. A. Ayoub et al., "Nicotinamide reduces infarction up to two hours after the onset of permanent focal cerebral ischemia in Wistar rats," Neurosci. Lett. 259(1), 21-24 (1999).

4. T. Mokudai et al., "Delayed treatment with nicotinamide (vitamin $B_{3}$ ) improves neurological outcome and reduces infarct volume after transient focal cerebral ischemia in Wistar rats," Stroke 31(7), 1679-1685 (2000).

5. Y. Sakakibara et al., "Post-treatment with nicotinamide (vitamin $B_{3}$ ) reduces the infarct volume following permanent focal cerebral ischemia in female Sprague-Dawley and Wistar rats," Neurosci. Lett. 281(2-3), $111-114$ (2000).

6. M. R. Hoane et al., "Nicotinamide reduces acute cortical neuronal death and edema in the traumatically injured brain," Neurosci. Lett. 408(1), 35-39 (2006)

7. M. R. Horsman, "Nicotinamide and other benzamide analogs as agents for overcoming hypoxic cell radiation resistance in tumours: a review," Acta. Oncol. 34(5), 571-587 (1995).

8. M. R. Horsman, D. J. Chaplin, and J. M. Brown, "Radiosensitization by nicotinamide in vivo: a greater enhancement of tumor damage compared to that of normal tissues," Radiat. Res. 109(3), 479-489 (1987).

9. M. R. Horsman et al., "The potentiation of radiation damage by nicotinamide in the SCCVII tumour in vivo," Radiother. Oncol. 18(1), 49-57 (1990).

10. M. R. Horsman et al., "Nicotinamide as a radiosensitizer in tumours and normal tissues: the importance of drug dose and timing," Radiother. Oncol. 45(2), 167-174 (1997).

11. D. J. Chaplin, M. R. Horsman, and M. J. Trotter, "Effect of nicotinamide on the microregional heterogeneity of oxygen delivery within a murine tumor," J. Natl. Cancer Inst. 82(8), 672-676 (1990).

12. S. A. Hill and D. J. Chaplin, "The effect of nicotinamide on microregional blood flow within tumours assessed using laser Doppler probes," Acta Oncol. 34(3), 401-404 (1995).

13. S. P. Robinson et al., "Effects of nicotinamide and carbogen on tumour oxygenation, blood flow, energetics and blood glucose levels," $\mathrm{Br}$. $J$. Cancer 82(12), 2007-2014 (2000).

14. D. K. Kelleher and P. W. Vaupel, "Nicotinamide exerts different acute effects on microcirculatory function and tissue oxygenation in rat tumors," Int. J. Radiat. Oncol. Biol. Phys. 26(1), 95-102 (1993).

15. T. Durduran et al., "Diffuse optics for tissue monitoring and tomography," Rep. Prog. Phys. 73(7), 076701 (2010).

16. C. Menon et al., "An integrated approach to measuring tumor oxygen status using human melanoma xenografts as a model," Cancer Res. 63(21), 7232-7240 (2003).

17. G. Yu et al., "Noninvasive monitoring of murine tumor blood flow during and after photodynamic therapy provides early assessment of therapeutic efficacy," Clin. Cancer Res. 11(9), 3543-3552 (2005).

18. E. M. Buckley et al., "Cerebral hemodynamics in preterm infants during positional intervention measured with diffuse correlation spectroscopy 
and transcranial Doppler ultrasound," Opt. Express 17(15), 1257112581 (2009)

19. P. Zirak et al., "Effects of acetazolamide on the micro- and macro-vascular cerebral hemodynamics: a diffuse optical and transcranial Doppler ultrasound study," Biomed. Opt. Express 1(5), 1443-1459 (2010).

20. N. Roche-Labarbe et al., "Noninvasive optical measures of $\mathrm{CBV}, \mathrm{StO}_{2}$, $\mathrm{CBF}$ index, and $\mathrm{rCMRO}_{2}$ in human premature neonates' brains in the first six weeks of life," Hum. Brain Mapp. 31(3), 341-352 (2010).

21. R. C. Mesquita et al., "Hemodynamic and metabolic diffuse optical monitoring in a mouse model of hindlimb ischemia," Biomed. Opt. Express 1(4), 1173-1187 (2010).

22. T. Durduran, "Non-invasive measurements of tissue hemodynamics with hybrid diffuse optical methods," Dissertation, University of Pennsylvania (2004).

23. T. Durduran et al., "Diffuse optical measurement of blood flow, blood oxygenation, and metabolism in a human brain during sensorimotor cortex activation," Opt. Lett. 29(15), 1766-1768 (2004).

24. G. Yu et al., "Validation of diffuse correlation spectroscopy for muscle blood flow with concurrent arterial spin labeled perfusion MRI," Opt. Express 15(3), 1064-1075 (2007).

25. S. A. Carp et al., "Validation of diffuse correlation spectroscopy measurements of rodent cerebral blood flow with simultaneous arterial spin labeling MRI; towards MRI-optical continuous cerebral metabolic monitoring," Biomed. Opt. Express 1(2), 553-565 (2010).

26. T. Durduran et al., "Optical measurement of cerebral hemodynamics and oxygen metabolism in neonates with congenital heart defects," J. Biomed. Opt. 15(3), 037004 (2010).

27. M. N. Kim et al., "Noninvasive measurement of cerebral blood flow and blood oxygenation using near-infrared and diffuse correlation spectroscopies in critically brain-injured adults," Neurocrit. Care 12(2), 173-180 (2010)

28. F. W. Prinzen and J. B. Bassingthwaighte, "Blood flow distributions by microsphere deposition methods," Cardiovasc. Res. 45(1), 13-21 (2000).

29. T. R. Cardinal and J. B. Hoying, "A modified fluorescent microspherebased approach for determining resting and hyperemic blood flows in individual murine skeletal muscles," Vascul. Pharmacol. 47(1), 48-56 (2007).

30. C. Zhou et al., "Diffuse optical monitoring of hemodynamic changes in piglet brain with closed head injury," J. Biomed. Opt. 14(3), 034015 (2009).

31. R. C. Mesquita et al., "Optical monitoring and detection of spinal cord ischemia," PLoS One 8(12), e83370 (2013).

32. A. S. Kogler et al., "Fiber-optic monitoring of spinal cord hemodynamics in experimental aortic occlusion," Anesthesiology 123(6), 13621373 (2015).

33. C. P. Reinhardt et al., "Stable labeled microspheres to measure perfusion: validation of a neutron activation assay technique," Am. J. Physiol. Heart Circ. Physiol. 280(1), H108-H116 (2001).

34. P. V. Turner et al., "Administration of substances to laboratory animals: routes of administration and factors to consider," J. Am. Assoc. Lab Anim. Sci. 50(5), 600-613 (2011).

35. R. W. Glenny, S. Bernard, and M. Brinkley, "Validation of fluorescentlabeled microspheres for measurement of regional organ perfusion," J. Appl. Physiol. 74(5), 2585-2597 (1993).

36. M. A. Serrat, "Measuring bone blood supply in mice using fluorescent microspheres," Nat. Protoc. 4(12), 1749-1758 (2009).

37. P. Farzam and T. Durduran, "Multidistance diffuse correlation spectroscopy for simultaneous estimation of blood flow index and optical properties," J. Biomed. Opt. 20(5), 055001 (2015).
38. S. L. Jacques, "Optical properties of biological tissues: a review," Phys. Med. Biol. 58(11), R37-R61 (2013).

39. H. Bay et al., "Speeded-up robust features (SURF)," Comput. Vis. Image Understanding 110(3), 346-359 (2008).

40. S. A. Eucker et al., "Development of a fluorescent microsphere technique for rapid histological determination of cerebral blood flow," Brain Res. 1326, 128-134 (2010).

41. S. P. Lloyd, "Least squares quantization in PCM," IEEE Trans. Inf. Theory 28(2), 129-137 (1982).

42. L. A. Sapirstein, "Regional blood flow by fractional distribution of indicators," Am. J. Physiol. 193(1), 161-168 (1958).

43. D. G. Hirst, B. Joiner, and V. K. Hirst, "Blood flow modification by nicotinamide and metoclopramide in mouse tumours growing in different sites," Br. J. Cancer 67(1), 1-6 (1993).

44. D. J. Honess and N. M. Bleehen, "Effects of the radiosensitising agent nicotinamide on relative tissue perfusion and kidney function in $\mathrm{C}_{3} \mathrm{H}$ mice," Radiother. Oncol. 27(2), 140-148 (1993).

45. R. M. Sheehan and E. M. Renkin, "Capillary, interstitial, and cell membrane barriers to blood-tissue transport of potassium and rubidium in mammalian skeletal muscle," Circ. Res. 30(5), 588-607 (1972).

46. D. O. Foster and M. L. Frydman, "Comparison of microspheres and ${ }^{86} \mathrm{Rb}^{+}$as tracers of the distribution of cardiac output in rats indicates invalidity of ${ }^{86} \mathrm{Rb}^{+}$-based measurements," Can. J. Physiol. Pharmacol. 56(1), 97-109 (1978).

47. R. W. Barbee et al., "Microsphere and dilution techniques for the determination of blood flows and volumes in conscious mice," Am. J. Physiol. 263(3 Pt. 2), R728-R733 (1992).

48. A. J. Maxwell et al., "Limb blood flow during exercise is dependent on nitric oxide," Circulation 98(4), 369-374 (1998).

49. R. E. Tomlinson and M. J. Silva, "Skeletal blood flow in bone repair and maintenance," Bone Res. 1(4), 311-322 (2013).

Ashley R. Proctor is a laboratory technician in the Department of Biomedical Engineering, University of Rochester. She earned her bachelor of science degree in neuroscience from Stonehill College in 2013.

Gabriel A. Ramirez is a laboratory technician in the Department of Biomedical Engineering, University of Rochester. He received his bachelor of science degree from Cornell University in civil and environmental engineering.

Songfeng Han is an optical scientist at Corning Inc. He received his $\mathrm{PhD}$ from the Institute of Optics at the University of Rochester in 2018.

Ziping Liu is an undergraduate student in the Department of Biomedical Engineering, University of Rochester. He is expecting to graduate with a bachelor of science in the Spring 2018.

Tracy M. Bubel is a technical associate II in the Center for Visual Science, University of Rochester. She earned her bachelor of science degree in marine science from the University of South Carolina.

Regine Choe is an associate professor in the Department of Biomedical Engineering, University of Rochester. Her current research focus is on the development, validation, and clinical translation of diffuse optical techniques for monitoring bone injury healing and breast cancer therapy. 